\title{
Broadband emission of blazar S5 $0716+714$ during impressive outbursts
}

\author{
Y. Kajiwara*1, M. Manganaro ${ }^{2}$ and E. Lindfors ${ }^{3}$ for the MAGIC Collaboration; \\ B. Rani ${ }^{4}$, S. G. Jorstad ${ }^{5,6}$, V. M. Larionov ${ }^{5,7}$, A. P. Marscher ${ }^{6}$ \\ ${ }^{1}$ Department of Physics, Kyoto University, 606-8502 Kyoto, Japan \\ ${ }^{2}$ University of Rijeka, Department of Physics, 51000 Rijeka, Croatia \\ ${ }^{3}$ Tuorla Observatory, Department of Physics and Astronomy, University of Turku, FI-20014 \\ Turku, Finland \\ ${ }^{4}$ NASA Goddard Space Flight Center, Greenbelt, MD 20771, USA \\ 5 Astronomical Institute, St. Petersburg State University, 199034, Russia \\ ${ }^{6}$ Institute for Astrophysical Research, Boston University, Boston MA 02215, USA \\ ${ }^{7}$ Pulkovo Observatory, 196140, St. Petersburg, Russia \\ E-mail: kajiwara.yuki.88rest.kyoto-u.ac.jp
}

In January 2015, an impressive outburst of the BL Lac object S5 0716+714 was registered in all energy bands, from low-frequency radio to very-high-energy gamma-rays (VHE, E > $100 \mathrm{GeV}$ ). For the first time for this object, simultaneous Fermi-LAT and MAGIC spectra were obtained, allowing a close investigation of the higher-energy peak of the spectral energy distribution (SED). The VLBA analysis of the parsec-scale jet, together with two-zone modeling of the simultaneous broadband SED, lead us to propose a scenario in which VHE emission arises during the entrance and exit of a superluminal knot through a recollimation shock in the inner jet. The gamma-ray emission in the high-energy $(0.1 \mathrm{GeV}<\mathrm{E}<100 \mathrm{GeV})$ and VHE bands is attributed to a shockshock interaction in the helical jet downstream of the mm-wave VLBI core, closely followed by optical and X-ray outbursts that appear to occur in the core. An interpretation that the VHE gamma-ray emission is associated with a jet component entering and exiting the core region is consistent with the timing of the two VHE gamma-ray emission peaks, optical polarization angle (EVPA) behavior, and jet kinematics: the first peak took place $\sim 2$ days after a very fast EVPA rotation, and the second $\sim 18$ days after the new knot emerged from the $43 \mathrm{GHz}$ VLBI core.

In December 2017 the source was detected by MAGIC at a flux level $\sim 6$ times higher than that of the 2015 outburst. We present the modeling of the 2015 flare and more recent results from the 2017 event.

36th International Cosmic Ray Conference -ICRC2019-

July 24th - August 1st, 2019

Madison, WI, U.S.A.

\footnotetext{
* Speaker.

†https://magic.mpp.mpg. de/. For collaboration list see PoS(ICRC2019)1177
} 


\section{S5 $0716+714$ Outburst in 2015}

S5 $0716+714$ is a BL Lac object featuring a peculiar rapid variability across the electromagnetic spectrum. This source, located at a redshift of $\mathrm{z}=0.3$, has been studied actively in all energy bands in past years, and it was discovered at very-high-energy gamma-ray (VHE, E > $100 \mathrm{GeV}$ ) in 2008 by MAGIC (Major Atmospheric Gamma-ray Imaging Cherenkov) [1]. At that time MAGIC consisted of a single telescope and the energy threshold for VHE gamma-rays was $\sim 400 \mathrm{GeV}$ in the zenith range of $47^{\circ}$ to $55^{\circ}$ in which the source was visible from the MAGIC site.

In January and February 2015, MAGIC detected an impressive outburst of S5 0716+714. The MAGIC observation was triggered by Fermi-LAT (Large Area Telescope), and consisted of the second detection of this source in the VHE range. In contrast to the 2008 detection, in 2015 the MAGIC system was working in stereo mode after a major upgrade, making it possible to reach an energy threshold of $\sim 125 \mathrm{GeV}$. Moreover, simultaneous multi-wavelength data from low radio to VHE gamma-rays including Very Long Baseline Interferometry (VLBI) and optical polarization were also taken, and spectral energy distributions (SEDs) from radio to VHE, including Fermi-LAT and NuSTAR data were for the first time obtained for this source [2].

\subsection{Multi-wavelength Observation}

Figure 1 shows the multi-wavelength light curves and optical polarization changes from the activity in January and February 2015. Two flaring states have been studied, indicated by the shaded areas in Figure 1: Phase A, from MJD 57040 to MJD 57050 (18th Jan to 27th Jan 2015) and Phase B, from MJD 57065 to MJD 57070 (12th Feb to 17th Feb 2015). Dashed vertical lines indicate the Gaussian fit peaks of the VHE gamma-ray emission in Phase A and B, respectively.

In Phase A, a broad-band flare from optical to VHE gamma-ray was seen, and a very rapid rotation $\left(\sim 360^{\circ}\right.$ in a day) of the electric vector position angle (EVPA) took place as shown in the bottom panel. The rotation started 4 days after the flare observed by Fermi-LAT in MJD 57038 (16th Jan 2015) which triggered VHE gamma-ray observations, and 2 days before the VHE gammaray highest flux detected peak (MJD 57047-25th Jan 2015). An EVPA swing was also seen in the first VHE detection of S5 0716+714 in 2008 [1, 3], but that time the change was slower and it took 6 days to rotate $\sim 320^{\circ}$, while in the present case the EVPA rotation was particularly rapid, happening overnight.

On the other hand, in Phase B flaring activities were observed in VHE gamma-rays and X-ray only, and this time no EVPA rotation was detected.

Very Long Baseline Array (VLBA) data of S5 0716+714 were obtained within the VLBA-BUBLAZAR program ${ }^{1}$. In figure 2 , color scale and contours indicate the polarized and total intensity respectively. The center of the radio core (the peak of the contour) is named A0 and assumed to be stationary, and the black horizontal line indicates its position. In addition to the radio core (A0), a stationary feature (A1), and two moving knots (K14a and K14b) have been identified.

The EVPA swing in the optical EVPA is explained in [2] as the passage of the superluminal $\mathrm{K} 14 \mathrm{~b}$ knot through the stationary feature A1 near the radio core. 


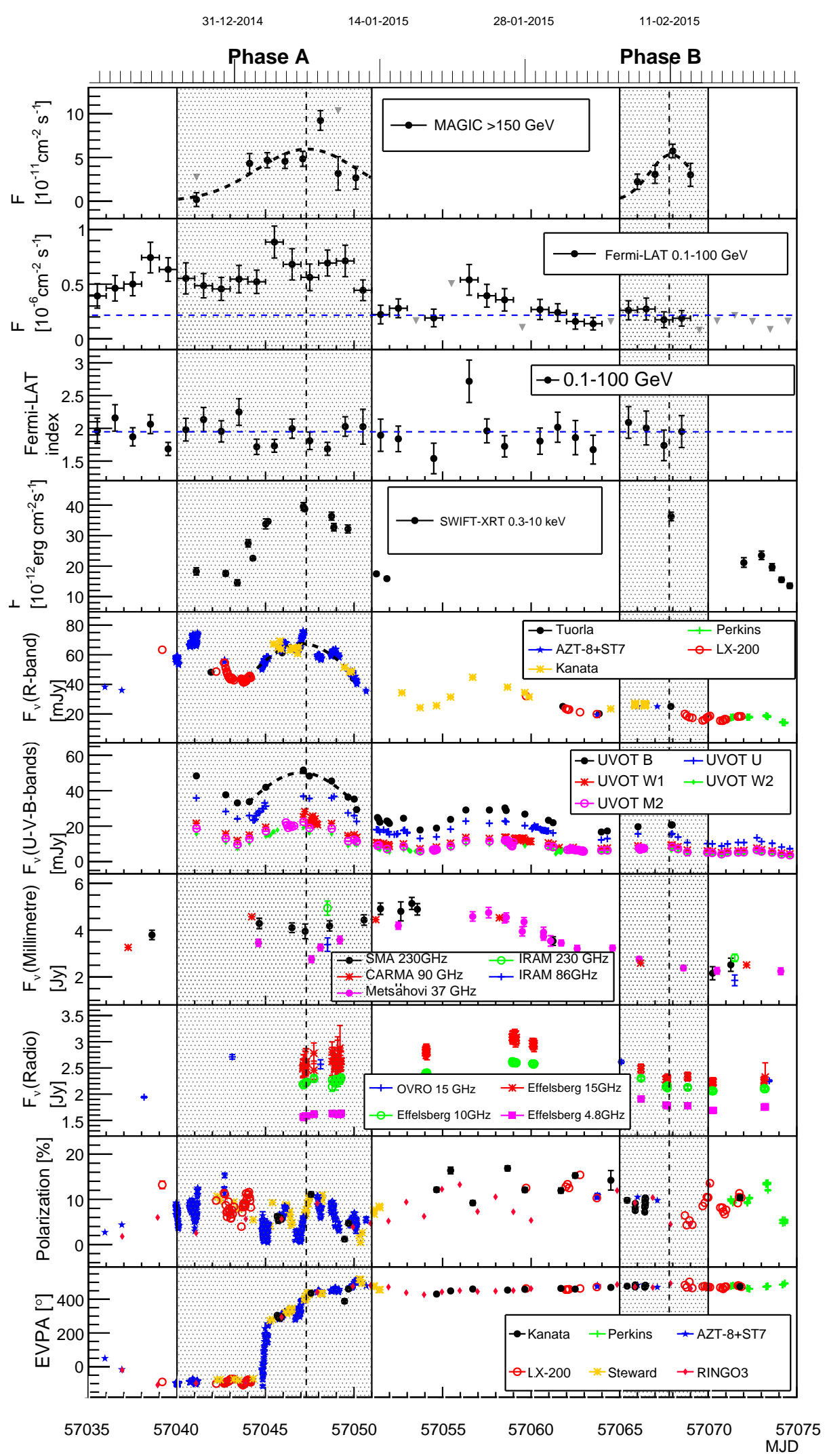

Figure 1: Multi-wavelength flux, photon index, and polarization curves of S5 0716+714 from MJD 57010 to MJD 57080. 


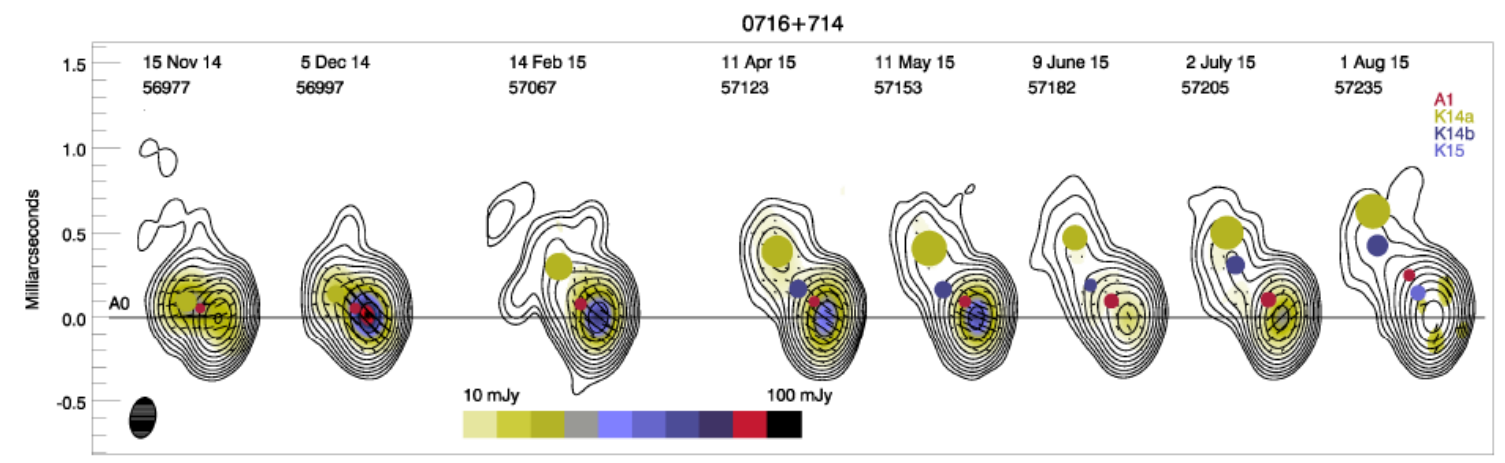

Figure 2: VLBI images of S5 0716+714 at $43 \mathrm{GHz}$. Color scale and contours indicate the polarized and total intensity, respectively.

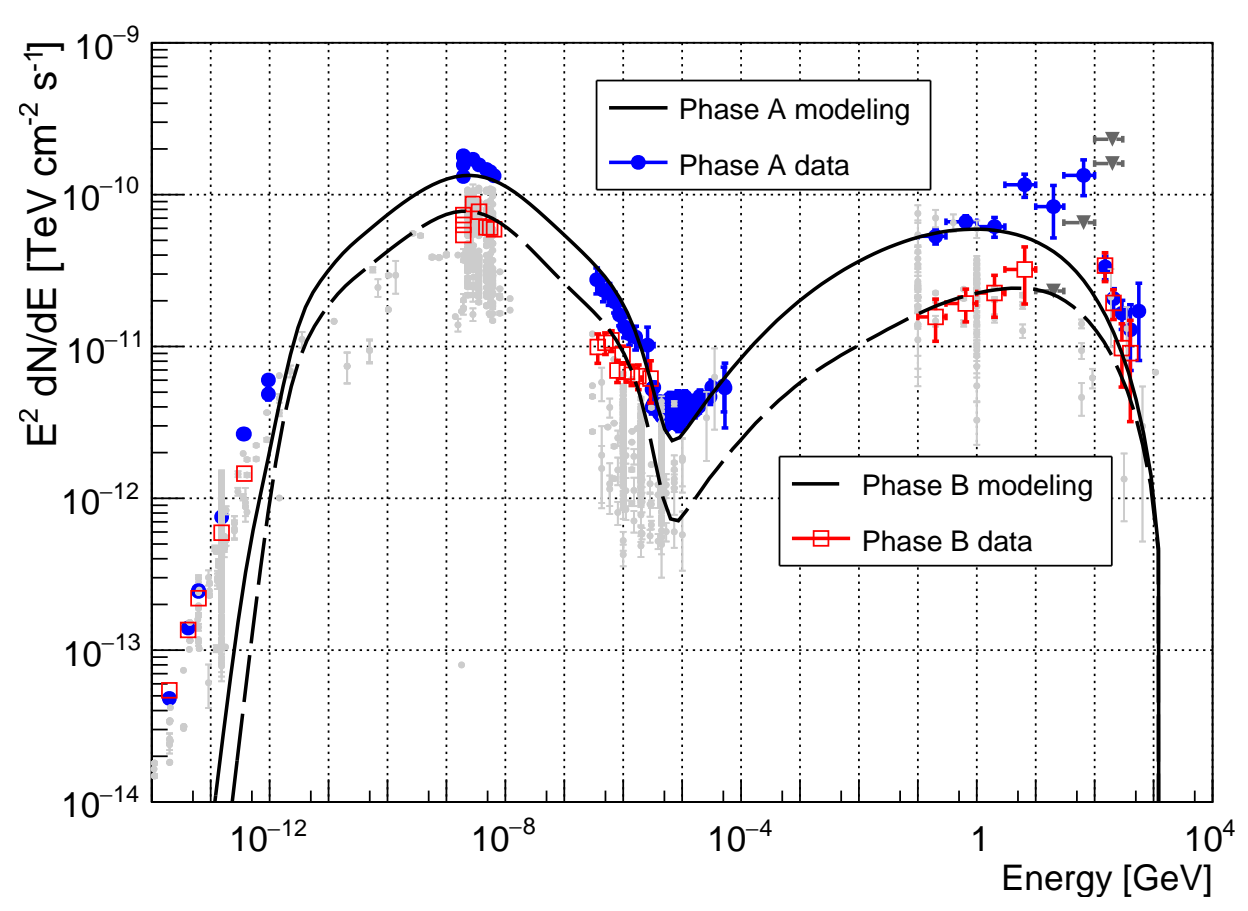

Figure 3: Broadband SED of S5 0716+714 for January and February 2015: represented data are from Phase A (full blue circles) and Phase B (open red squares). The full and dashed lines indicate the two-zone modelings of the two Phases, respectively. Archival data from ASDC are shown in gray.

\subsection{Modeling}

Figure 3 shows the multi-wavelength SED of S5 0716+714 during Phase A (full blue circles) and Phase B (open red squares). Archival data from ASDC ${ }^{2}$ are shown in grey.

Phase A is clearly an unprecedentedly high state of the source with respect to archival data,

\footnotetext{
${ }^{1}$ www.bu.edu/blazars/VLBAproject.html

${ }^{2}$ http://www.asdc.asi.it/
} 
in all the energy bands. Differently from the modeling of the data in [1], a simple one-zone synchrotron self-Compton (SSC) model could not explain the observed SED. Instead, a two-zone model has been adopted, in which a superluminal knot (blob 1) is interacting with a recollimation shock region (blob 2). The total of the two components (the black lines in Figure 3, full for Phase A and dashed for Phase B) and their interaction reconstructed the broadband SED. The presence of NuSTAR data constrained with high precision the synchrotron peak and the valley between the two humps: the IC bump of the SED, though, is not as well described by the model, especially in the VHE gamma-ray part: a slight difference in the 10-100 GeV range between the model line and observed plots is visible in Figure 3. Such a sharp feature of the higher-energy bump could be explained by external seed photon injection, but for this source there is no observational evidence for external photons.

The gap between the model and data might have been caused because the model was too simplified and did not include details like the geometry of magnetic fields. The measured fast EVPA rotation cannot currently be included in the modeling: only few models can describe broadband SED together with polarization effects, as for instance the TEMZ model [4].

Nevertheless in [2] conclusions can be drawn regarding the emission scenario: the flaring multi-wavelength activity period during Phase A coincides with the passage of a moving feature through a stationary feature at $\sim 0.1$ mas, while the VHE gamma-ray flares coincide with in the entrance and exit of a superluminal knot in and out of a recollimation shock in the inner jet. This suggests that moving shock - stationary shock interaction in the jet is responsible for the observed flares and EVPA swing.

The jet behavior, studied with VLBA-BU-BLAZAR data, is in agreement with the scenario described in [5], suggesting a connection between jet kinematics and the observed broadband flaring activity. More precisely, the gamma-ray emission in the HE and VHE bands is attributed to a shock in the helical jet downstream of the core, closely followed by an optical and X-ray outburst in the core. The presence of low radio activity, observed during Phase A, was not reported in April 2008 when MAGIC observed the source for the first time in the VHE range [1]. In this case it could be a delayed response of a previous, less intense flare, resembling the behavior of the same source in the radio band reported in [6,7], when simultaneous optical and/or gamma flares lagged behind the radio counterparts by almost two months.

\section{New Results}

In December 2017, a new flare of S5 0716+714 has been observed in VHE gamma-rays by MAGIC. This flare lasted only for 2 nights, but was detected with intensity 3 times stronger than the 2015 flare [8]. Figure 4a shows the distribution of the squared angular distance $\theta^{2}$ between the reconstructed source position and the nominal source position (points) or the background estimation position (shaded area) for S5 $0716+714$. The total effective time after quality cuts for the 2 nights is 3.81 hours, and the Li\&Ma significance is $31.43 \sigma$.

The shaded area represents the distribution of the squared angular distances $\left(\theta^{2}\right)$ between the background estimation position (OFF) and the reconstructed event directions. The points indicate the $\theta^{2}$ between the reconstructed event directions and the nominal source position in the sky (ON). The number of gamma-ray events (excess) is obtained by subtracting the background from the total 


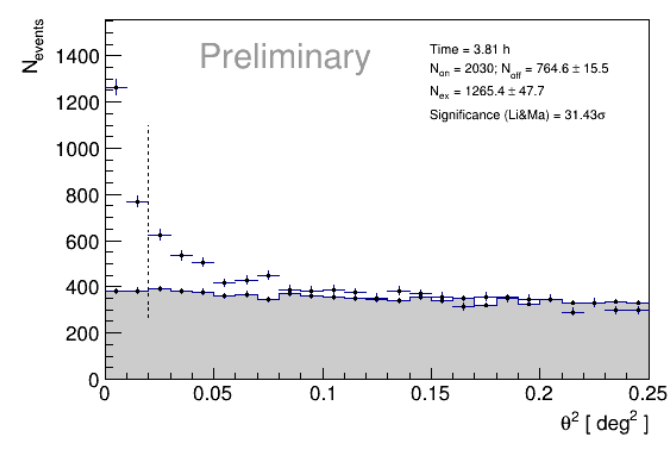

(a) Distribution of the squared angular distance, $\theta^{2}$, between the reconstructed source position and the nominal source position (points) or the background estimation position (shaded area) for S5 0716+714: The vertical dashed line shows the value of $\theta^{2}$ up to which the number of excess events and significance are computed.

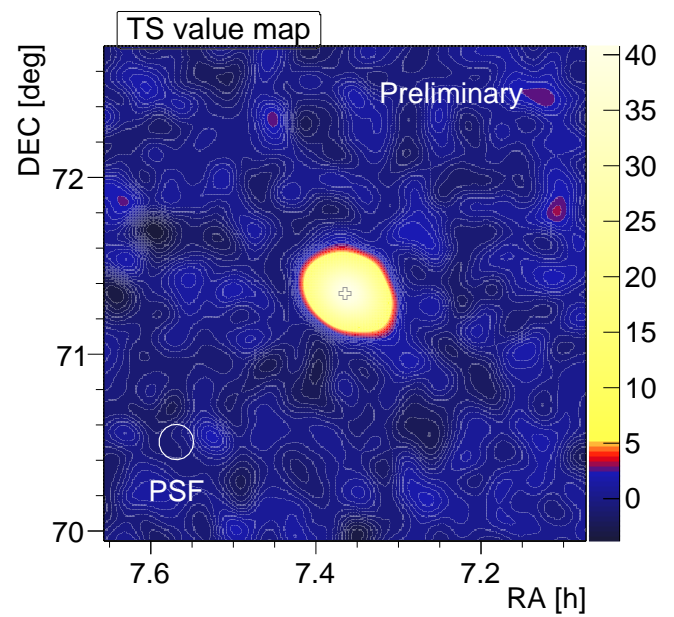

(b) Test Statistics Skymap of S5 0716+714. The color scale represents the significance.

Figure 4: Latest results for VHE gamma-ray observations of S50716+714: $\theta^{2}$ distribution and skymap for December 2017 data.

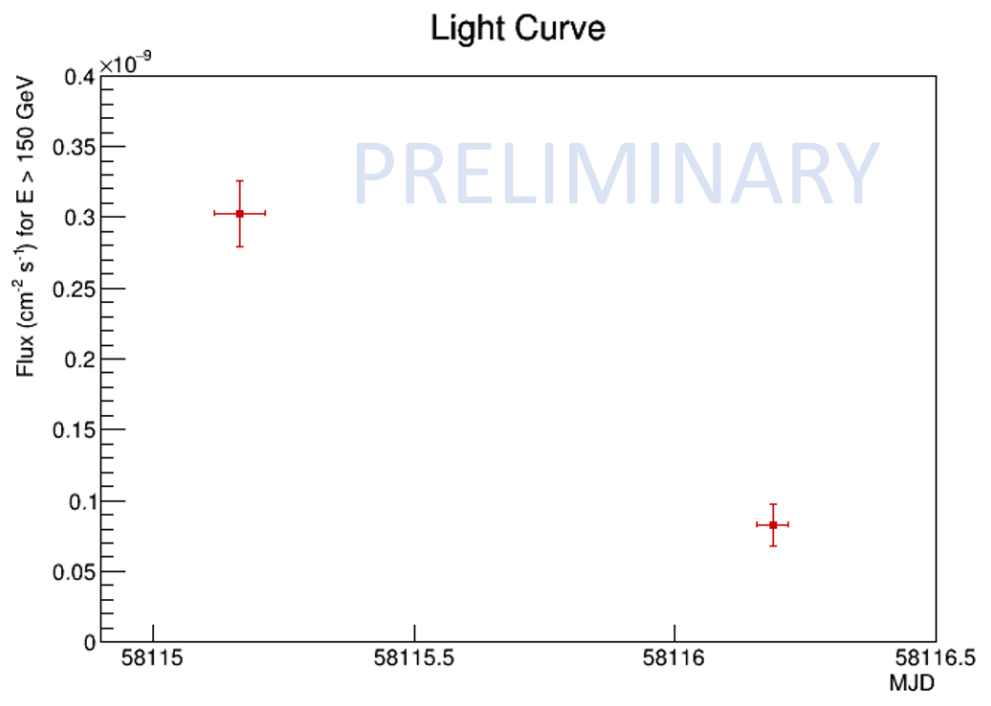

Figure 5: Nigthly lightcurve of VHE gamma-rays for the S5 0716+714 flare in December 2017. 
number of events and applying a cut in the $\theta^{2}$ distribution, indicated by the vertical dashed line in Figure 4a.

Figure $4 \mathrm{~b}$ shows the Test Statistics (TS) skymap of the source. For the TS we adopted eq. 17 of [9], applied on a smoothed and modeled background estimation. Its null hypothesis distribution mostly resembles a Gaussian function, but in general can have a somewhat different shape or width. The nightly light curve for this latest collected data is shown in Figure 5. After a preliminary analysis and quality cuts, the flux above $150 \mathrm{GeV}$ detected in the night MJD 58115 (28th Dec 2017) was $(2.63 \pm 0.19) \times 10^{-10} \mathrm{~cm}^{-2} \mathrm{~s}^{-1}$, while the second night, MJD 58116 (29th Dec 2017) it was significantly decreased to $(8.21 \pm 1.36) \times 10^{-11} \mathrm{~cm}^{-2} \mathrm{~s}^{-1}$.

A study of the source during this high flux activity in the VHE gamma-ray together with available multi-wavelength data is in preparation for the MAGIC Collaboration.

\section{Acknowledgements}

MAGIC Collaboration: https://magic.mpp.mpg.de/acknowledgments_ICRC2019/

The VLBA is an instrument of the Long Baseline Observatory. The Long Baseline Observatory is a facility of the National Science Foundation operated by Associated Universities, Inc.

The research at St. Petersburg University was supported by Russian Science Foundation grant 1712-01029. The research at Boston University was supported in part by National Science Foundation grant AST-1615796 and NASA Fermi Guest Investigator grant 80NSSC17K0649.

\section{References}

[1] MAGIC Collaboration, H. Anderhub et al., Discovery of Very High Energy $\gamma$-rays from the blazar S5 0716+714, Astrophys. J. 704 (2009) L129, [0 907 . 2386].

[2] MAGIC Collaboration, M. L. Ahnen, S. Ansoldi, L. A. Antonelli, C. Arcaro, D. Baack, A. Babić, B. Banerjee, P. Bangale, and U. Barres de Almeida, Multi-wavelength characterization of the blazar S5 0716+714 during an unprecedented outburst phase, 619 (Nov, 2018) A45, [1807.00413].

[3] “Atel \#1502.” http://www.astronomerstelegram.org/?findmsg, 2008.

[4] A. P. Marscher, Turbulent, Extreme Multi-Zone Model for Simulating Flux and Polarization Variability in Blazars, Astrophys. J. 780 (2014) 87, [1311. 7665].

[5] B. Rani, T. P. Krichbaum, A. P. Marscher, J. A. Hodgson, L. Fuhrmann, E. Angelakis, S. Britzen, and J. A. Zensus, Connection between inner jet kinematics and broadband flux variability in the BL Lacertae object S5 0716+714, 578 (Jun, 2015) A123, [1503.04218].

[6] B. Rani, T. P. Krichbaum, L. Fuhrmann, M. Böttcher, B. Lott, H. D. Aller, M. F. Aller, E. Angelakis, U. Bach, and D. Bastieri, Radio to gamma-ray variability study of blazar S5 0716+714, 552 (Apr, 2013) A11, [1301.7087].

[7] B. Rani, T. P. Krichbaum, A. P. Marscher, S. G. Jorstad, J. A. Hodgson, L. Fuhrmann, and J. A. Zensus, Jet outflow and gamma-ray emission correlations in S5 0716+714, 571 (Nov, 2014) L2, [1410.0196].

[8] “Atel \#11100.” http://www.astronomerstelegram.org/?read=11100, 2017. 
[9] T. P. Li and Y. Q. Ma, Analysis methods for results in gamma-ray astronomy., 272 (Sep, 1983) $317-324$. 